\title{
LATTICE REPRESENTATIONS OF HEISENBERG GROUPS
}

\author{
JAE-HYUN YANG
}

\section{Introduction}

For any positive integers $g$ and $h$, we consider the Heisenberg group

$$
H_{\mathbb{R}}^{(g, h)}:=\left\{(\lambda, \mu, \kappa) \mid \lambda, \mu \in \mathbb{R}^{(h, g)}, \kappa \in \mathbb{R}^{(h, h)}, \kappa+\mu^{t} \lambda \text { symmetric }\right\} .
$$

Recall that the multiplication law is

$$
(\lambda, \mu, \kappa) \circ\left(\lambda^{\prime}, \mu^{\prime}, \kappa^{\prime}\right):=\left(\lambda+\lambda^{\prime}, \mu+\mu^{\prime}, \kappa+\kappa^{\prime}+\lambda^{t} \mu^{\prime}-\mu^{t} \lambda^{\prime}\right) .
$$

Here $\mathbb{R}^{(h, g)}\left(\right.$ resp. $\left.\mathbb{R}^{(h, h)}\right)$ denotes the set of all $h \times g($ resp. $h \times h)$ real matrices.

The Heisenberg group $H_{\mathbb{R}}^{(g, h)}$ is embedded to the symplectic group $S p(g+h, \mathbb{R})$ via the mapping

$$
H_{\mathbb{R}}^{(g, h)} \ni(\lambda, \mu, \kappa) \longmapsto\left(\begin{array}{cccc}
E_{g} & 0 & 0 & { }^{t} \mu \\
\lambda & E_{h} & \mu & \kappa \\
0 & 0 & E_{g} & -{ }^{t} \lambda \\
0 & 0 & 0 & E_{h}
\end{array}\right) \in S p(g+h, \mathbb{R}) .
$$

This Heisenberg group is a 2-step nilpotent Lie group and is important in the study of toroidal compactifications of Siegel moduli spaces. In fact, $H_{\mathbb{R}}^{(g, h)}$ is obtained as the unipotent radical of the parabolic subgroup of $S p(g+h, \mathbb{R})$ associated with the rational boundary component $F_{g}$ (cf. [F-C] p. 123 or [N] p. 21). For the motivation of the study of this Heisenberg group we refer to [Y4]-[Y8] and [Z]. We refer to [Y1]-[Y3] for more results on $H_{\mathbb{R}}^{(g, h)}$.

This work was partially supported by the Max-Planck-Institut für Mathematik and TGRCKOSEF.

Keywords : Heisenberg groups, Schrödinger representations, lattice representations, theta functions

Typeset by $\mathcal{A} \mathcal{M} \mathcal{S}-\mathrm{T}_{\mathrm{E}} \mathrm{X}$ 
In $[\mathrm{C}]$, P. Cartier stated without proof that for $h=1$, the lattice representation of $H_{\mathbb{R}}^{(g, 1)}$ associated to the lattice $L$ is unitarily equivalent to the direct sum of $\left[L^{*}: L\right]^{\frac{1}{2}}$ copies of the Schrödinger representation of $H_{\mathbb{R}}^{(g, 1)}$, where $L^{*}$ is the dual lattice of $L$ with respect to a certain nondegenerate alternating bilinear form. R. Berndt proved that the above fact for the case $h=1$ in his lecture notes [B]. In this paper, we give a complete proof of Cartier's theorem for $H_{\mathbb{R}}^{(g, h)}$.

Main Theorem. Let $\mathcal{M}$ be a positive definite, symmetric half-integral matrix of degree $h$ and $L$ be a self-dual lattice in $\mathbb{C}^{(h, g)}$. Then the lattice representation $\pi_{\mathcal{M}}$ of $H_{\mathbb{R}}^{(g, h)}$ associated with $L$ and $\mathcal{M}$ is unitarily equivalent to the direct sum of $(\operatorname{det} 2 \mathcal{M})^{g}$ copies of the Schrödinger representation of $H_{\mathbb{R}}^{(g, h)}$. For more details, we refer to Section 3.

The paper is organized as follows. In Section 2, we review the Schrödinger representations of the Heisenberg group $H_{\mathbb{R}}^{(g, h)}$. In Section 3 , we prove the main theorem. In the final section, we provide a relation between lattice representations and theta functions.

ACKNowledgement. This work was in part done during my stay at the MaxPlanck-Institut für Mathematik in Bonn. I am very grateful to the institute for hospitality and financial support. I also would like to give my hearty thanks to the Department of Mathematics at Harvard University for its hospitality during my short stay in Cambridge.

Notations: We denote by $\mathbb{Z}, \mathbb{R}$ and $\mathbb{C}$ the ring of integers, the field of real numbers, and the field of complex numbers respectively. The symbol $\mathbb{C}_{1}^{\times}$denotes the multiplicative group consisting of all complex numbers $z$ with $|z|=1$, and the symbol $S p(g, \mathbb{R})$ the symplectic group of degree $g, H_{g}$ the Siegel upper half plane of degree $g$. The symbol ":=" means that the expression on the right hand side is the definition of that on the left. We denote by $\mathbb{Z}^{+}$the set of all positive integers, by $F^{(k, l)}$ the set of all $k \times l$ matrices with entries in a commutative ring $F$. For any $M \in F^{(k, l)},{ }^{t} M$ denotes the transpose matrix of $M$. For $A \in F^{(k, k)}, \sigma(A)$ denotes the trace of $A$. For $A \in F^{(k, l)}$ and $B \in F^{(k, k)}$, we set $B[A]={ }^{t} A B A$. We denote the identity matrix of degree $k$ by $E_{k}$. For a positive integer $n, \operatorname{Symm}(n, K)$ denotes the vector space consisting of all symmetric $n \times n$ matrices with entries in a field $K$.

\section{SCHRÖDINGER REPRESENTATIONS}

First of all, we observe that $H_{\mathbb{R}}^{(g, h)}$ is a 2-step nilpotent Lie group. It is easy 
to see that the inverse of an element $(\lambda, \mu, \kappa) \in H_{\mathbb{R}}^{(g, h)}$ is given by

$$
(\lambda, \mu, \kappa)^{-1}=\left(-\lambda,-\mu,-\kappa+\lambda^{t} \mu-\mu^{t} \lambda\right) .
$$

Now we set

$$
[\lambda, \mu, \kappa]:=(0, \mu, \kappa) \circ(\lambda, 0,0)=\left(\lambda, \mu, \kappa-\mu^{t} \lambda\right)
$$

Then $H_{\mathbb{R}}^{(g, h)}$ may be regarded as a group equipped with the following multiplication

$$
[\lambda, \mu, \kappa] \diamond\left[\lambda_{0}, \mu_{0}, \kappa_{0}\right]:=\left[\lambda+\lambda_{0}, \mu+\mu_{0}, \kappa+\kappa_{0}+\lambda^{t} \mu_{0}+\mu_{0}{ }^{t} \lambda\right] .
$$

The inverse of $[\lambda, \mu, \kappa] \in H_{\mathbb{R}}^{(g, h)}$ is given by

$$
[\lambda, \mu, \kappa]^{-1}=\left[-\lambda,-\mu,-\kappa+\lambda^{t} \mu+\mu^{t} \lambda\right]
$$

We set

$$
K:=\left\{[0, \mu, \kappa] \in H_{\mathbb{R}}^{(g, h)} \mid \mu \in \mathbb{R}^{(h, g)}, \kappa={ }^{t} \kappa \in \mathbb{R}^{(h, h)}\right\} .
$$

Then $K$ is a commutative normal subgroup of $H_{\mathbb{R}}^{(g, h)}$. Let $\hat{K}$ be the Pontrajagin dual of $K$, i.e., the commutative group consisting of all unitary characters of $K$. Then $\hat{K}$ is isomorphic to the additive group $\mathbb{R}^{(h, g)} \times \operatorname{Symm}(h, \mathbb{R})$ via

$$
<a, \hat{a}>:=e^{2 \pi i \sigma\left(\hat{\mu}^{t} \mu+\hat{\kappa} \kappa\right)}, \quad a=[0, \mu, \kappa] \in K, \hat{a}=(\hat{\mu}, \hat{\kappa}) \in \hat{K} .
$$

We put

$$
S:=\left\{[\lambda, 0,0] \in H_{\mathbb{R}}^{(g, h)} \mid \lambda \in \mathbb{R}^{(h, g)}\right\} \cong \mathbb{R}^{(h, g)} .
$$

Then $S$ acts on $K$ as follows:

$$
\alpha_{\lambda}([0, \mu, \kappa]):=\left[0, \mu, \kappa+\lambda^{t} \mu+\mu^{t} \lambda\right], \quad[\lambda, 0,0] \in S .
$$

It is easy to see that the Heisenberg group $\left(H_{\mathbb{R}}^{(g, h)}, \diamond\right)$ is isomorphic to the semidirect product $S \ltimes K$ of $S$ and $K$ whose multiplication is given by

$$
(\lambda, a) \cdot\left(\lambda_{0}, a_{0}\right):=\left(\lambda+\lambda_{0}, a+\alpha_{\lambda}\left(a_{0}\right)\right), \quad \lambda, \lambda_{0} \in S, a, a_{0} \in K .
$$

On the other hand, $S$ acts on $\hat{K}$ by

$$
\alpha_{\lambda}^{*}(\hat{a}):=(\hat{\mu}+2 \hat{\kappa} \lambda, \hat{\kappa}), \quad[\lambda, 0,0] \in S, \quad a=(\hat{\mu}, \hat{\kappa}) \in \hat{K} .
$$

Then, we have the relation $\left\langle\alpha_{\lambda}(a), \hat{a}>=<a, \alpha_{\lambda}^{*}(\hat{a})>\right.$ for all $a \in K$ and $\hat{a} \in \hat{K}$. 
We have two types of $S$-orbits in $\hat{K}$.

Type I. Let $\hat{\kappa} \in \operatorname{Symm}(h, \mathbb{R})$ with $\hat{\kappa} \neq 0$. The $S$-orbit of $\hat{a}(\hat{\kappa}):=(0, \hat{\kappa}) \in \hat{K}$ is given by

$$
\hat{\mathcal{O}}_{\hat{\kappa}}:=\left\{(2 \hat{\kappa} \lambda, \hat{\kappa}) \in \hat{K} \mid \lambda \in \mathbb{R}^{(h, g)}\right\} \cong \mathbb{R}^{(h, g)} .
$$

Type II. Let $\hat{y} \in \mathbb{R}^{(h, g)}$. The $S$-orbit $\hat{\mathcal{O}}_{\hat{y}}$ of $\hat{a}(\hat{y}):=(\hat{y}, 0)$ is given by

$$
\hat{\mathcal{O}}_{\hat{y}}:=\{(\hat{y}, 0)\}=\hat{a}(\hat{y}) .
$$

We have

$$
\hat{K}=\left(\bigcup_{\hat{\kappa} \in \operatorname{Symm}(h, \mathbb{R})} \hat{\mathcal{O}}_{\hat{\kappa}}\right) \bigcup\left(\bigcup_{\hat{y} \in \mathbb{R}^{(h, g)}} \hat{\mathcal{O}}_{\hat{y}}\right)
$$

as a set. The stabilizer $S_{\hat{\kappa}}$ of $S$ at $\hat{a}(\hat{\kappa})=(0, \hat{\kappa})$ is given by

$$
S_{\hat{\kappa}}=\{0\} .
$$

And the stabilizer $S_{\hat{y}}$ of $S$ at $\hat{a}(\hat{y})=(\hat{y}, 0)$ is given by

$$
S_{\hat{y}}=\left\{[\lambda, 0,0] \mid \lambda \in \mathbb{R}^{(h, g)}\right\}=S \cong \mathbb{R}^{(h, g)} .
$$

From now on, we set $G:=H_{\mathbb{R}}^{(g, h)}$ for brevity. It is known that $K$ is a closed, commutative normal subgroup of $G$. Since $(\lambda, \mu, \kappa)=\left(0, \mu, \kappa+\mu^{t} \lambda\right) \circ(\lambda, 0,0)$ for $(\lambda, \mu, \kappa) \in G$, the homogeneous space $X:=K \backslash G$ can be identified with $\mathbb{R}^{(h, g)}$ via

$$
K g=K \circ(\lambda, 0,0) \longmapsto \lambda, \quad g=(\lambda, \mu, \kappa) \in G .
$$

We observe that $G$ acts on $X$ by

$$
(K g) \cdot g_{0}:=K\left(\lambda+\lambda_{0}, 0,0\right)=\lambda+\lambda_{0},
$$

where $g=(\lambda, \mu, \kappa) \in G$ and $g_{0}=\left(\lambda_{0}, \mu_{0}, \kappa_{0}\right) \in G$.

If $g=(\lambda, \mu, \kappa) \in G$, we have

$$
k_{g}=\left(0, \mu, \kappa+\mu^{t} \lambda\right), \quad s_{g}=(\lambda, 0,0)
$$

in the Mackey decomposition of $g=k_{g} \circ s_{g}$ ( cf. [M] ). Thus if $g_{0}=\left(\lambda_{0}, \mu_{0}, \kappa_{0}\right) \in G$, then we have

$$
s_{g} \circ g_{0}=(\lambda, 0,0) \circ\left(\lambda_{0}, \mu_{0}, \kappa_{0}\right)=\left(\lambda+\lambda_{0}, \mu_{0}, \kappa_{0}+\lambda^{t} \mu_{0}\right)
$$


and so

$$
k_{s_{g} \circ g_{0}}=\left(0, \mu_{0}, \kappa_{0}+\mu_{0}{ }^{t} \lambda_{0}+\lambda^{t} \mu_{0}+\mu_{0}{ }^{t} \lambda\right) .
$$

For a real symmetric matrix $c={ }^{t} c \in \mathbb{R}^{(h, h)}$ with $c \neq 0$, we consider the one-dimensional unitary representation $\sigma_{c}$ of $K$ defined by

$$
\sigma_{c}((0, \mu, \kappa)):=e^{2 \pi i \sigma(c \kappa)} I, \quad(0, \mu, \kappa) \in K,
$$

where $I$ denotes the identity mapping. Then the induced representation $U\left(\sigma_{c}\right):=$ $\operatorname{Ind}_{K}^{G} \sigma_{c}$ of $G$ induced from $\sigma_{c}$ is realized in the Hilbert space $\mathcal{H}_{\sigma_{c}}=L^{2}(X, d \dot{g}, \mathbb{C}) \cong$ $L^{2}\left(\mathbb{R}^{(h, g)}, d \xi\right)$ as follows. If $g_{0}=\left(\lambda_{0}, \mu_{0}, \kappa_{0}\right) \in G$ and $x=K g \in X$ with $g=(\lambda, \mu, \kappa) \in G$, we have

$$
\left(U_{g_{0}}\left(\sigma_{c}\right) f\right)(x)=\sigma_{c}\left(k_{s_{g} \circ g_{0}}\right)\left(f\left(x g_{0}\right)\right), \quad f \in \mathcal{H}_{\sigma_{c}} .
$$

It follows from (2.15) that

$$
\left(U_{g_{0}}\left(\sigma_{c}\right) f\right)(\lambda)=e^{2 \pi i \sigma\left\{c\left(\kappa_{0}+\mu_{0}{ }^{t} \lambda_{0}+2 \lambda^{t} \mu_{0}\right)\right\}} f\left(\lambda+\lambda_{0}\right) .
$$

Here, we identified $x=K g$ (resp. $\left.x g_{0}=K g g_{0}\right)$ with $\lambda\left(\right.$ resp. $\left.\lambda+\lambda_{0}\right)$. The induced representation $U\left(\sigma_{c}\right)$ is called the Schrödinger representation of $G$ associated with $\sigma_{c}$. Thus $U\left(\sigma_{c}\right)$ is a monomial representation.

Now, we denote by $\mathcal{H}^{\sigma_{c}}$ the Hilbert space consisting of all functions $\phi: G \longrightarrow \mathbb{C}$ which satisfy the following conditions:

(1) $\phi(g)$ is measurable with respect to $d g$,

(2) $\phi((0, \mu, \kappa) \circ g))=e^{2 \pi i \sigma(c \kappa)} \phi(g)$ for all $g \in G$,

(3) $\|\phi\|^{2}:=\int_{X}|\phi(g)|^{2} d \dot{g}<\infty, \quad \dot{g}=K g$,

where $d g($ resp. $d \dot{g})$ is a $G$-invariant measure on $G$ (resp. $X=K \backslash G$ ). The inner product $($,$) on \mathcal{H}^{\sigma_{c}}$ is given by

$$
\left(\phi_{1}, \phi_{2}\right):=\int_{G} \phi_{1}(g) \overline{\phi_{2}(g)} d g \quad \text { for } \phi_{1}, \phi_{2} \in \mathcal{H}^{\sigma_{c}} \text {. }
$$

We observe that the mapping $\Phi_{c}: \mathcal{H}_{\sigma_{c}} \longrightarrow \mathcal{H}^{\sigma_{c}}$ defined by

$$
\left(\Phi_{c}(f)\right)(g):=e^{2 \pi i \sigma\left\{c\left(\kappa+\mu^{t} \lambda\right)\right\}} f(\lambda), \quad f \in \mathcal{H}_{\sigma_{c}}, g=(\lambda, \mu, \kappa) \in G
$$

is an isomorphism of Hilbert spaces. The inverse $\Psi_{c}: \mathcal{H}^{\sigma_{c}} \longrightarrow \mathcal{H}_{\sigma_{c}}$ of $\Phi_{c}$ is given by

$$
\left(\Psi_{c}(\phi)\right)(\lambda):=\phi((\lambda, 0,0)), \quad \phi \in \mathcal{H}^{\sigma_{c}}, \lambda \in \mathbb{R}^{(h, g)} .
$$


The Schrödinger representation $U\left(\sigma_{c}\right)$ of $G$ on $\mathcal{H}^{\sigma_{c}}$ is given by

$$
\left(U_{g_{0}}\left(\sigma_{c}\right) \phi\right)(g)=e^{2 \pi i \sigma\left\{c\left(\kappa_{0}+\mu_{0}{ }^{t} \lambda_{0}+\lambda^{t} \mu_{0}-\lambda_{0}{ }^{t} \mu\right)\right\}} \phi\left(\left(\lambda_{0}, 0,0\right) \circ g\right),
$$

where $g_{0}=\left(\lambda_{0}, \mu_{0}, \kappa_{0}\right), g=(\lambda, \mu, \kappa) \in G$ and $\phi \in \mathcal{H}^{\sigma_{c}} \cdot(2.21)$ can be expressed as follows.

$$
\left(U_{g_{0}}\left(\sigma_{c}\right) \phi\right)(g)=e^{2 \pi i \sigma\left\{c\left(\kappa_{0}+\kappa+\mu_{0}{ }^{t} \lambda_{0}+\mu^{t} \lambda+2 \lambda^{t} \mu_{0}\right)\right\}} \phi\left(\left(\lambda_{0}+\lambda, 0,0\right)\right) .
$$

Theorem 2.1. Let $c$ be a positive symmetric half-integral matrix of degree $h$. Then the Schrödinger representation $U\left(\sigma_{c}\right)$ of $G$ is irreducible.

Proof. The proof can be found in [Y1], theorem 3.

\section{Proof of the Main Theorem}

Let $L:=\mathbb{Z}^{(h, g)} \times \mathbb{Z}^{(h, g)}$ be the lattice in the vector space $V \cong \mathbb{C}^{(h, g)}$. Let $B$ be an alternating bilinear form on $V$ such that $B(L, L) \subset \mathbb{Z}$, that is, $\mathbb{Z}$-valued on $L \times L$. The dual $L_{B}^{*}$ of $L$ with respect to $B$ is defined by

$$
L_{B}^{*}:=\{v \in V \mid B(v, l) \in \mathbb{Z} \text { for all } l \in L\} .
$$

Then $L \subset L_{B}^{*}$. If $B$ is nondegenerate, $L_{B}^{*}$ is also a lattice in $V$, called the dual lattice of $L$. In case $B$ is nondegenerate, there exist a $\mathbb{Z}$-basis $\left\{\xi_{11}, \xi_{12}, \cdots, \xi_{h g}, \eta_{11}, \eta_{12}, \cdots\right.$, $\left.\eta_{h g}\right\}$ of $L$ and a set $\left\{e_{11}, e_{12}, \cdots, e_{h g}\right\}$ of positive integers such that $e_{11}\left|e_{12}, e_{12}\right| e_{13}$, $\cdots, e_{h, g-1} \mid e_{h g}$ for which

$$
\left(\begin{array}{cc}
B\left(\xi_{k a}, \xi_{l b}\right) & B\left(\xi_{k a}, \eta_{l b}\right) \\
B\left(\eta_{k a}, \xi_{l b}\right) & B\left(\eta_{k a}, \eta_{l b}\right.
\end{array}\right)=\left(\begin{array}{cc}
0 & e \\
-e & 0
\end{array}\right)
$$

where $1 \leq k, l \leq h, 1 \leq a, b \leq g$ and $e:=\operatorname{diag}\left(e_{11}, e_{12}, \cdots, e_{h g}\right)$ is the diagonal matrix of degree $h g$ with entries $e_{11}, e_{12}, \cdots, e_{h g}$. It is well known that $\left[L_{B}^{*}: L\right]=$ $(\operatorname{det} e)^{2}=\left(e_{11} e_{12} \cdots e_{h g}\right)^{2}$ (cf. [I] p. 72). The number det $e$ is called the Pfaffian of $B$.

Now, we consider the following subgroups of $G$ :

$$
\Gamma_{L}:=\left\{(\lambda, \mu, \kappa) \in G \mid(\lambda, \mu) \in L, \kappa \in \mathbb{R}^{(h, h)}\right\}
$$

and

$$
\Gamma_{L_{B}^{*}}:=\left\{(\lambda, \mu, \kappa) \in G \mid(\lambda, \mu) \in L_{B}^{*}, \kappa \in \mathbb{R}^{(h, h)}\right\}
$$


Then both $\Gamma_{L}$ and $\Gamma_{L_{B}^{*}}$ are normal subgroups of $G$. We set

$$
\mathcal{Z}_{0}:=\left\{(0,0, \kappa) \in G \mid \kappa={ }^{t} \kappa \in \mathbb{Z}^{(h, h)} \text { integral }\right\} .
$$

It is easy to show that

$$
\Gamma_{L_{B}^{*}}=\left\{g \in G \mid g \gamma g^{-1} \gamma^{-1} \in \mathcal{Z}_{0} \text { for all } \gamma \in \Gamma_{L}\right\} .
$$

We define

$$
Y_{L}:=\left\{\varphi \in \operatorname{Hom}\left(\Gamma_{L}, \mathbb{C}_{1}^{\times}\right) \mid \varphi \text { is trivial on } \mathcal{Z}_{0}\right\}
$$

and

$$
Y_{L, S}:=\left\{\varphi \in Y_{L} \mid \varphi(\kappa)=e^{2 \pi i \sigma(S \kappa)} \text { for all } \kappa={ }^{t} \kappa \in \mathbb{R}^{(h, h)}\right\}
$$

for each symmetric real matrix $S$ of degree $h$. We observe that, if $S$ is not halfintegral, then $Y_{L}=\emptyset$ and so $Y_{L, S}=\emptyset$. It is clear that, if $S$ is symmetric halfintegral, then $Y_{L, S}$ is not empty.

Thus we have

$$
Y_{L}=\cup_{\mathcal{M}} Y_{L, \mathcal{M}}
$$

where $\mathcal{M}$ runs through the set of all symmetric half-integral matrices of degree $h$.

Lemma 3.1. Let $\mathcal{M}$ be a symmetric half-integral matrix of degree $h$ with $\mathcal{M} \neq 0$. Then any element $\varphi$ of $Y_{L, \mathcal{M}}$ is of the form $\varphi_{\mathcal{M}, q}$. Here $\varphi_{\mathcal{M}, q}$ is the character of $\Gamma_{L}$ defined by

$$
\varphi_{\mathcal{M}, q}((l, \kappa)):=e^{2 \pi i \sigma(\mathcal{M} \kappa)} \cdot e^{\pi i q(l)} \quad \text { for }(l, \kappa) \in \Gamma_{L},
$$

where $q: L \longrightarrow \mathbb{R} / 2 \mathbb{Z} \cong[0,2)$ is a function on $L$ satisfying the following condition:

$$
q\left(l_{0}+l_{1}\right) \equiv q\left(l_{0}\right)+q\left(l_{1}\right)-2 \sigma\left\{\mathcal{M}\left(\lambda_{0}{ }^{t} \mu_{1}-\mu_{0}{ }^{t} \lambda_{1}\right)\right\} \quad(\bmod 2)
$$

for all $l_{0}=\left(\lambda_{0}, \mu_{0}\right) \in L$ and $l_{1}=\left(\lambda_{1}, \mu_{1}\right) \in L$.

Proof. (3.8) follows immediately from the fact that $\varphi_{\mathcal{M}, q}$ is a character of $\Gamma_{L}$. It is obvious that any element of $Y_{L, \mathcal{M}}$ is of the form $\varphi_{\mathcal{M}, q}$.

Lemma 3.2. An element of $Y_{L, 0}$ is of the form $\varphi_{k, l}\left(k, l \in \mathbb{R}^{(h, g)}\right)$. Here $\varphi_{k, l}$ is the character of $\Gamma_{L}$ defined by

$$
\varphi_{k, l}(\gamma):=e^{2 \pi i \sigma\left(k^{t} \lambda+l^{t} \mu\right)}, \quad \gamma=(\lambda, \mu, \kappa) \in \Gamma_{L} .
$$

Proof. It is easy to prove and so we omit the proof. 
Lemma 3.3. Let $\mathcal{M}$ be a nonsingular symmetric half-integral matrix of degree $h$. Let $\varphi_{\mathcal{M}, q_{1}}$ and $\varphi_{\mathcal{M}, q_{2}}$ be the characters of $\Gamma_{L}$ defined by (3.7). The character $\varphi$ of $\Gamma_{L}$ defined by $\varphi:=\varphi_{\mathcal{M}, q_{1}} \cdot \varphi_{\mathcal{M}, q_{2}}^{-1}$ is an element of $Y_{L, 0}$.

Proof. It follows from the existence of an element $g=\left(\mathcal{M}^{-1} \lambda, \mathcal{M}^{-1} \mu, 0\right) \in G$ with $(\lambda, \mu) \in V$ such that

$$
\varphi_{\mathcal{M}, q_{1}}(\gamma)=\varphi_{\mathcal{M}, q_{2}}\left(g \gamma g^{-1}\right) \text { for all } \gamma \in \Gamma_{L}
$$

For a unitary character $\varphi_{\mathcal{M}, q}$ of $\Gamma_{L}$ defined by (3.7), we let

$$
\pi_{\mathcal{M}, q}:=\operatorname{Ind}_{\Gamma_{L}}^{G} \varphi_{\mathcal{M}, q}
$$

be the representation of $G$ induced from $\varphi_{\mathcal{M}, q}$. Let $\mathcal{H}_{\mathcal{M}, q}$ be the Hilbert space consisting of all measurable functions $\phi: G \longrightarrow \mathbb{C}$ satisfying

(L1) $\phi(\gamma g)=\varphi_{\mathcal{M}, q}(\gamma) \phi(g)$ for all $\gamma \in \Gamma_{L}$ and $g \in G$.

(L2) $\|\phi\|_{\mathcal{M}, q}^{2}:=\int_{\Gamma_{L} \backslash G}|\phi(\bar{g})|^{2} d \bar{g}<\infty, \quad \bar{g}=\Gamma_{L} g$.

The induced representation $\pi_{\mathcal{M}, q}$ is realized in $\mathcal{H}_{\mathcal{M}, q}$ as follows:

$$
\left(\pi_{\mathcal{M}, q}\left(g_{0}\right) \phi\right)(g):=\phi\left(g g_{0}\right), \quad g_{0}, g \in G, \phi \in \mathcal{H}_{\mathcal{M}, q}
$$

The representation $\pi_{\mathcal{M}, q}$ is called the lattice representation of $G$ associated with the lattice $L$.

Main Theorem. Let $\mathcal{M}$ be a positive definite, symmetric half integral matrix of degree $h$. Let $\varphi_{\mathcal{M}}$ be the character of $\Gamma_{L}$ defined by $\varphi_{\mathcal{M}}((\lambda, \mu, \kappa)):=e^{2 \pi i \sigma(\mathcal{M} \kappa)}$ for all $(\lambda, \mu, \kappa) \in \Gamma_{L}$. Then the lattice representation

$$
\pi_{\mathcal{M}}:=\operatorname{Ind}_{\Gamma_{L}}^{G} \varphi_{\mathcal{M}}
$$

induced from the character $\varphi_{\mathcal{M}}$ is unitarily equivalent to the direct sum

$$
\bigoplus U\left(\sigma_{\mathcal{M}}\right):=\bigoplus \operatorname{Ind}_{K}^{G} \sigma_{\mathcal{M}} \quad\left((\operatorname{det} 2 \mathcal{M})^{g} \text {-copies }\right)
$$

of the Schrödinger representation $\operatorname{Ind}_{K}^{G} \sigma_{\mathcal{M}}$. 
Proof. We first recall that the induced representation $\pi_{\mathcal{M}}$ is realized in the Hilbert space $\mathcal{H}_{\mathcal{M}}$ consisting of all measurable functions $\phi: G \longrightarrow \mathbb{C}$ satisfying the conditions

$$
\phi\left(\left(\lambda_{0}, \mu_{0}, \kappa_{0}\right) \circ g\right)=e^{2 \pi i \sigma\left(\mathcal{M} \kappa_{0}\right)} \phi(g), \quad\left(\lambda_{0}, \mu_{0}, \kappa_{0}\right) \in \Gamma_{L}, g \in G
$$

and

$$
\|\phi\|_{\pi, \mathcal{M}}^{2}:=\int_{\Gamma_{L} \backslash G}|\phi(\bar{g})|^{2} d \bar{g}<\infty, \quad \bar{g}=\Gamma_{L} \circ g .
$$

Now, we write

$$
g_{0}=\left[\lambda_{0}, \mu_{0}, \kappa_{0}\right] \in \Gamma_{L} \text { and } g=[\lambda, \mu, \kappa] \in G
$$

For $\phi \in \mathcal{H}_{\mathcal{M}}$, we have

$$
\phi\left(g_{0} \diamond g\right)=\phi\left(\left[\lambda_{0}+\lambda, \mu_{0}+\mu, \kappa_{0}+\kappa+\lambda_{0}{ }^{t} \mu+\mu^{t} \lambda_{0}\right]\right) .
$$

On the other hand, we get

$$
\begin{aligned}
\phi\left(g_{0} \diamond g\right) & =\phi\left(\left(\lambda_{0}, \mu_{0}, \kappa_{0}-\mu_{0}{ }^{t} \lambda_{0}\right) \circ g\right) \\
& =e^{2 \pi i \sigma\left\{\mathcal{M}\left(\kappa_{0}-\mu_{0}{ }^{t} \lambda_{0}\right)\right\}} \phi(g) \\
& =e^{2 \pi i \sigma\left(\mathcal{M} \kappa_{0}\right)} \phi(g) \quad\left(\text { because } \sigma\left(\mathcal{M} \mu_{0}{ }^{t} \lambda_{0}\right) \in \mathbb{Z}\right)
\end{aligned}
$$

Thus, putting $\kappa^{\prime}:=\kappa_{0}+\lambda_{0}{ }^{t} \mu+\mu^{t} \lambda_{0}$, we get

$$
\phi\left(\left[\lambda_{0}+\lambda, \mu_{0}+\mu, \kappa+\kappa^{\prime}\right]\right)=e^{2 \pi i \sigma\left(\mathcal{M} \kappa^{\prime}\right)} \cdot e^{-4 \pi i \sigma\left(\mathcal{M} \lambda_{0}{ }^{t} \mu\right)} \phi([\lambda, \mu, \kappa]) .
$$

Putting $\lambda_{0}=\kappa_{0}=0$ in $(3.16)$, we have

$$
\phi\left(\left[\lambda, \mu+\mu_{0}, \kappa\right]\right)=\phi([\lambda, \mu, \kappa]) \text { for all } \mu_{0} \in \mathbb{Z}^{(h, g)} \text { and }[\lambda, \mu, \kappa] \in G \text {. }
$$

Therefore if we fix $\lambda$ and $\kappa, \phi$ is periodic in $\mu$ with respect to the lattice $\mathbb{Z}^{(h, g)}$ in $\mathbb{R}^{(h, g)}$. We note that

$$
\phi([\lambda, \mu, \kappa])=\phi([0,0, \kappa] \diamond[\lambda, \mu, 0])=e^{2 \pi i \sigma(\mathcal{M} \kappa)} \phi([\lambda, \mu, 0])
$$

for $[\lambda, \mu, \kappa] \in G$. Hence, $\phi$ admits a Fourier expansion in $\mu$ :

$$
\phi([\lambda, \mu, \kappa])=e^{2 \pi i \sigma(\mathcal{M} \kappa)} \sum_{N \in \mathbb{Z}^{(h, g)}} c_{N}(\lambda) e^{2 \pi i \sigma\left(N^{t} \mu\right)} .
$$


If $\lambda_{0} \in \mathbb{Z}^{(h, g)}$, then we have

$$
\begin{aligned}
\phi\left(\left[\lambda+\lambda_{0}, \mu, \kappa\right]\right) & =e^{2 \pi i \sigma(\mathcal{M} \kappa)} \sum_{N \in \mathbb{Z}^{(h, g)}} c_{N}\left(\lambda+\lambda_{0}\right) e^{2 \pi i \sigma\left(N^{t} \mu\right)} \\
& =e^{-4 \pi i \sigma\left(\mathcal{M} \lambda_{0}{ }^{t} \mu\right)} \phi([\lambda, \mu, \kappa]) \quad(\text { by }(3.16)) \\
& =e^{-4 \pi i \sigma\left(\mathcal{M} \lambda_{0}{ }^{t} \mu\right)} e^{2 \pi i \sigma(\mathcal{M} \kappa)} \sum_{N \in \mathbb{Z}^{(h, g)}} c_{N}(\lambda) e^{2 \pi i \sigma\left(N^{t} \mu\right)}, \\
& =e^{2 \pi i \sigma(\mathcal{M} \kappa)} \sum_{N \in \mathbb{Z}^{(h, g)}} c_{N}(\lambda) e^{2 \pi i \sigma\left\{\left(N-2 \mathcal{M} \lambda_{0}\right)^{t} \mu\right\}} . \quad \text { by }
\end{aligned}
$$

So we get

$$
\begin{aligned}
& \sum_{N \in \mathbb{Z}^{(h, g)}} c_{N}\left(\lambda+\lambda_{0}\right) e^{2 \pi i \sigma\left(N^{t} \mu\right)} \\
= & \sum_{N \in \mathbb{Z}^{(h, g)}} c_{N}(\lambda) e^{2 \pi i \sigma\left\{\left(N-2 \mathcal{M} \lambda_{0}\right)^{t} \mu\right\}} \\
= & \sum_{N \in \mathbb{Z}^{(h, g)}} c_{N+2 \mathcal{M} \lambda_{0}}(\lambda) e^{2 \pi i \sigma\left(N^{t} \mu\right)} .
\end{aligned}
$$

Hence, we get

$$
c_{N}\left(\lambda+\lambda_{0}\right)=c_{N+2 \mathcal{M} \lambda_{0}}(\lambda) \text { for all } \lambda_{0} \in \mathbb{Z}^{(h, g)} \text { and } \lambda \in \mathbb{R}^{(h, g)} .
$$

Consequently, it is enough to know only the coefficients $c_{\alpha}(\lambda)$ for the representatives $\alpha$ in $\mathbb{Z}^{(h, g)}$ modulo $2 \mathcal{M}$. It is obvious that the number of all such $\alpha$ 's is $(\operatorname{det} 2 \mathcal{M})^{g}$. We denote by $\mathcal{J}$ a complete system of such representatives in $\mathbb{Z}^{(h, g)}$ modulo $2 \mathcal{M}$.

Then, we have

$$
\begin{aligned}
\phi([\lambda, \mu, \kappa])=e^{2 \pi i \sigma(\mathcal{M} \kappa)} & \left\{\sum_{N \in \mathbb{Z}^{(h, g)}} c_{\alpha+2 \mathcal{M N N}}(\lambda) e^{2 \pi i \sigma\left\{(\alpha+2 \mathcal{M} N)^{t} \mu\right\}}\right. \\
& +\sum_{N \in \mathbb{Z}^{(h, g)}} c_{\beta+2 \mathcal{M N}}(\lambda) e^{2 \pi i \sigma\left\{(\beta+2 \mathcal{M} N)^{t} \mu\right\}} \\
& \cdot \\
& \cdot \\
& \left.+\sum_{N \in \mathbb{Z}^{(h, g)}} c_{\gamma+2 \mathcal{M N}}(\lambda) e^{2 \pi i\left\{(\gamma+2 \mathcal{M} N)^{t} \mu\right\}}\right\},
\end{aligned}
$$

where $\{\alpha, \beta, \cdots, \gamma\}$ denotes the complete system $\mathcal{J}$. 
For each $\alpha \in \mathcal{J}$, we denote by $\mathcal{H}_{\mathcal{M}, \alpha}$ the Hilbert space consisting of Fourier expansions

$$
e^{2 \pi i \sigma(\mathcal{M} \kappa)} \sum_{N \in \mathbb{Z}^{(h, g)}} c_{\alpha+2 \mathcal{M} N}(\lambda) e^{2 \pi i \sigma\left\{(\alpha+2 \mathcal{M} N)^{t} \mu\right\}}, \quad(\lambda, \mu, \kappa) \in G,
$$

where $c_{N}(\lambda)$ denotes the coefficients of the Fourier expansion (3.18) of $\phi \in \mathcal{H}_{\mathcal{M}}$ and $\phi$ runs over the set $\left\{\phi \in \pi_{\mathcal{M}}\right\}$. It is easy to see that $\mathcal{H}_{\mathcal{M}, \alpha}$ is invariant under $\pi_{\mathcal{M}}$. We denote the restriction of $\pi_{\mathcal{M}}$ to $\mathcal{H}_{\mathcal{M}, \alpha}$ by $\pi_{\mathcal{M}, \alpha}$. Then we have

$$
\pi_{\mathcal{M}}=\bigoplus_{\alpha \in \mathcal{J}} \pi_{\mathcal{M}, \alpha}
$$

Let $\phi_{\alpha} \in \pi_{\mathcal{M}, \alpha}$. Then for $[\lambda, \mu, \kappa] \in G$, we get

$$
\phi_{\alpha}([\lambda, \mu, \kappa])=e^{2 \pi i \sigma(\mathcal{M} \kappa)} \sum_{N \in \mathbb{Z}^{(h, g)}} c_{\alpha+2 \mathcal{M N}}(\lambda) e^{2 \pi i \sigma\left\{(\alpha+2 \mathcal{M} N)^{t} \mu\right\}} .
$$

We put

$$
I_{\lambda}:=\overbrace{[0,1] \times[0,1] \times \cdots \times[0,1]}^{(h \times g) \text {-times }} \subset\left\{[\lambda, 0,0] \mid \lambda \in \mathbb{R}^{(h, g)}\right\}
$$

and

$$
I_{\mu}:=\overbrace{[0,1] \times[0,1] \times \cdots \times[0,1]}^{(h \times g) \text {-times }} \subset\left\{[0, \mu, 0] \mid \mu \in \mathbb{R}^{(h, g)}\right\} .
$$

Then, we obtain

$$
\int_{I_{\mu}} \phi_{\alpha}([\lambda, \mu, \kappa]) e^{-2 \pi i \sigma\left(\alpha^{t} \mu\right)} d \mu=e^{2 \pi i \sigma(\mathcal{M} \kappa)} c_{\alpha}(\lambda), \quad \alpha \in \mathcal{J} .
$$

Since $\Gamma_{L} \backslash G \cong I_{\lambda} \times I_{\mu}$, we get

$$
\begin{aligned}
\left\|\phi_{\alpha}\right\|_{\pi, \mathcal{M}, \alpha}^{2}: & =\left\|\phi_{\alpha}\right\|_{\pi, \mathcal{M}}^{2}=\int_{\Gamma_{L} \backslash G}\left|\phi_{\alpha}(\bar{g})\right|^{2} d \bar{g} \\
& =\int_{I_{\lambda}} \int_{I_{\mu}}\left|\phi_{\alpha}(\bar{g})\right|^{2} d \lambda d \mu \\
& =\int_{I_{\lambda} \times I_{\mu}}\left|\sum_{N \in \mathbb{Z}^{(h, g)}} c_{\alpha+2 \mathcal{M} N}(\lambda) e^{2 \pi i \sigma\left\{(\alpha+2 \mathcal{M} N)^{t} \mu\right\}}\right|^{2} d \lambda d \mu \\
& =\int_{I_{\lambda}} \sum_{N \in \mathbb{Z}^{(h, g)}}\left|c_{\alpha+2 \mathcal{M N}}(\lambda)\right|^{2} d \lambda \quad \sum_{I_{\lambda}} \sum_{N \in \mathbb{Z}^{(h, g)}}\left|c_{\alpha}(\lambda+N)\right|^{2} d \lambda \quad(\text { by (3.19) ) } \\
& =\int_{\mathbb{R}^{(h, g)}}\left|c_{\alpha}(\lambda)\right|^{2} d \lambda .
\end{aligned}
$$


Since $\phi_{\alpha} \in \pi_{\mathcal{M}, \alpha},\left\|\phi_{\alpha}\right\|_{\pi, \mathcal{M}, \alpha}<\infty$ and so $c_{\alpha}(\lambda) \in L^{2}\left(\mathbb{R}^{(h, g)}, d \xi\right)$ for all $\alpha \in \mathcal{J}$.

For each $\alpha \in \mathcal{J}$, we define the mapping $\vartheta_{\mathcal{M}, \alpha}$ on $L^{2}\left(\mathbb{R}^{(h, g)}, d \xi\right)$ by

$$
\left(\vartheta_{\mathcal{M}, \alpha} f\right)([\lambda, \mu, \kappa]):=e^{2 \pi i \sigma(\mathcal{M} \kappa)} \sum_{N \in \mathbb{Z}^{(h, g)}} f(\lambda+N) e^{2 \pi i \sigma\left\{(\alpha+2 \mathcal{M} N)^{t} \mu\right\}}
$$

where $f \in L^{2}\left(\mathbb{R}^{(h, g)}, d \xi\right)$ and $[\lambda, \mu, \kappa] \in G$.

Lemma 3.4. For each $\alpha \in \mathcal{J}$, the image of $L^{2}\left(\mathbb{R}^{(h, g)}, d \xi\right)$ under $\vartheta_{\mathcal{M}, \alpha}$ is contained in $\mathcal{H}_{\mathcal{M}, \alpha}$. Moreover, the mapping $\vartheta_{\mathcal{M}, \alpha}$ is a one-to-one unitary operator of $L^{2}\left(\mathbb{R}^{(h, g)}, d \xi\right)$ onto $\mathcal{H}_{\mathcal{M}, \alpha}$ preserving the norms. In other words, the mapping

$$
\vartheta_{\mathcal{M}, \alpha}: L^{2}\left(\mathbb{R}^{(h, g)}, d \xi\right) \longrightarrow \mathcal{H}_{\mathcal{M}, \alpha}
$$

is an isometry.

Proof. We already showed that $\vartheta_{\mathcal{M}, \alpha}$ preserves the norms. First, we observe that if $\left(\lambda_{0}, \mu_{0}, \kappa_{0}\right) \in \Gamma_{L}$ and $g=[\lambda, \mu, \kappa] \in G$,

$$
\begin{aligned}
\left(\lambda_{0}, \mu_{0}, \kappa_{0}\right) \circ g & =\left[\lambda_{0}, \mu_{0}, \kappa_{0}+\mu_{0}{ }^{t} \lambda_{0}\right] \diamond[\lambda, \mu, \kappa] \\
& =\left[\lambda_{0}+\lambda, \mu_{0}+\mu, \kappa+\kappa_{0}+\mu_{0}{ }^{t} \lambda_{0}+\lambda_{0}{ }^{t} \mu+\mu^{t} \lambda_{0}\right] .
\end{aligned}
$$

Thus we get

$$
\begin{aligned}
& \left(\vartheta_{\mathcal{M}, \alpha} f\right)\left(\left(\lambda_{0}, \mu_{0}, \kappa_{0}\right) \circ g\right) \\
& =e^{2 \pi i \sigma\left\{\mathcal{M}\left(\kappa+\kappa_{0}+\mu_{0}{ }^{t} \lambda_{0}+\lambda_{0}{ }^{t} \mu+\mu^{t} \lambda_{0}\right)\right\}} \sum_{N \in \mathbb{Z}^{(h, g)}} f\left(\lambda+\lambda_{0}+N\right) e^{2 \pi i\left\{(\alpha+2 \mathcal{M} N)^{t}\left(\mu_{0}+\mu\right)\right\}} \\
& =e^{2 \pi i \sigma\left(\mathcal{M} \kappa_{0}\right)} \cdot e^{2 \pi i \sigma(\mathcal{M} \kappa)} \cdot e^{2 \pi i \sigma\left(\alpha^{t} \mu_{0}\right)} \sum_{N \in \mathbb{Z}^{(h, g)}} f(\lambda+N) e^{2 \pi i \sigma\left\{(\alpha+2 \mathcal{M} N)^{t} \mu\right\}} \\
& =e^{2 \pi i \sigma\left(\mathcal{M} \kappa_{0}\right)}\left(\vartheta_{\mathcal{M}, \alpha} f\right)(g) .
\end{aligned}
$$

Here, in the above equalities we used the facts that $2 \sigma\left(\mathcal{M} N^{t} \mu_{0}\right) \in \mathbb{Z}$ and $\alpha^{t} \mu_{0} \in$ $\mathbb{Z}$. It is easy to show that

$$
\int_{\Gamma_{L} \backslash G}\left|\vartheta_{\mathcal{M}, \alpha} f(\bar{g})\right|^{2} d \bar{g}=\int_{\mathbb{R}^{(h, g)}}|f(\lambda)|^{2} d \lambda=\|f\|_{2}^{2}<\infty .
$$

This completes the proof of Lemma 3.4. 
Finally, it is easy to show that for each $\alpha \in \mathcal{J}$, the mapping $\vartheta_{\mathcal{M}, \alpha}$ intertwines the Schrödinger representation $\left(U\left(\sigma_{\mathcal{M}}\right), L^{2}\left(\mathbb{R}^{(h, g)}, d \xi\right)\right)$ and the representation $\left(\pi_{\mathcal{M}, \alpha}, \mathcal{H}_{\mathcal{M}, \alpha}\right)$. Therefore, by Lemma 3.4 , for each $\alpha \in \mathcal{J}, \pi_{\mathcal{M}, \alpha}$ is unitarily equivalent to $U\left(\sigma_{\mathcal{M}}\right)$ and so $\pi_{\mathcal{M}, \alpha}$ is an irreducible unitary representation of $G$. According to (3.20), the induced representation $\pi_{\mathcal{M}}$ is unitarily equivalent to

$$
\bigoplus U\left(\sigma_{\mathcal{M}}\right) \quad\left((\operatorname{det} 2 \mathcal{M})^{g} \text {-copies }\right)
$$

This completes the proof of the Main Theorem.

\section{Relation of Lattice Representations to Theta Functions}

In this section, we state the connection between lattice representations and theta functions. As before, we write $V=\mathbb{R}^{(h, g)} \times \mathbb{R}^{(h, g)} \cong \mathbb{C}^{(h, g)}, L=\mathbb{Z}^{(h, g)} \times \mathbb{Z}^{(h, g)}$ and $\mathcal{M}$ is a positive symmetric half-integral matrix of degree $h$. The function $q_{\mathcal{M}}: L \longrightarrow \mathbb{R} / 2 \mathbb{Z}=[0,2)$ defined by

$$
q_{\mathcal{M}}((\xi, \eta)):=2 \sigma\left(\mathcal{M} \xi^{t} \eta\right), \quad(\xi, \eta) \in L
$$

satisfies Condition (3.8). We let $\varphi_{\mathcal{M}, q_{\mathcal{M}}}: \Gamma_{L} \longrightarrow \mathbb{C}_{1}^{\times}$be the character of $\Gamma_{L}$ defined by

$$
\varphi_{\mathcal{M}, q_{\mathcal{M}}}((l, \kappa))=e^{2 \pi i \sigma(\mathcal{M} \kappa)} e^{\pi i q_{\mathcal{M}}(l)}, \quad(l, \kappa) \in \Gamma_{L} .
$$

We denote by $\mathcal{H}_{\mathcal{M}, q_{\mathcal{M}}}$ the Hilbert space consisting of measurable functions $\phi$ : $G \longrightarrow \mathbb{C}$ which satisfy Condition (4.2) and Condition (4.3):

$$
\begin{aligned}
\phi((l, \kappa) \circ g)= & \varphi_{\mathcal{M}, q_{\mathcal{M}}}((l, \kappa)) \phi(g) \quad \text { for all }(l, \kappa) \in \Gamma_{L} \text { and } g \in G . \\
& \int_{\Gamma_{L} \backslash G}\|\phi(\dot{g})\|^{2} d \dot{g}<\infty, \quad \dot{g}=\Gamma_{L} \circ g .
\end{aligned}
$$

Then the lattice representation

$$
\pi_{\mathcal{M}, q_{\mathcal{M}}}:=\operatorname{Ind}_{\Gamma_{L}}^{G} \varphi_{\mathcal{M}, q_{\mathcal{M}}}
$$

of $G$ induced from the character $\varphi_{\mathcal{M}, q_{\mathcal{M}}}$ is realized in $\mathcal{H}_{\mathcal{M}, q_{\mathcal{M}}}$ as

$$
\left(\pi_{\mathcal{M}, q_{\mathcal{M}}}\left(g_{0}\right) \phi\right)(g)=\phi\left(g g_{0}\right), \quad g_{0}, g \in G, \phi \in \mathcal{H}_{\mathcal{M}, q_{\mathcal{M}}}
$$

Let $\mathbf{H}_{\mathcal{M}, q_{\mathcal{M}}}$ be the vector space consisting of measurable functions $F: V \longrightarrow \mathbb{C}$ satisfying Conditions (4.4) and (4.5).

$$
F(\lambda+\xi, \mu+\eta)=e^{2 \pi i \sigma\left\{\mathcal{M}\left(\xi^{t} \eta+\lambda^{t} \eta-\mu^{t} \xi\right)\right\}} F(\lambda, \mu)
$$

for all $(\lambda, \mu) \in V$ and $(\xi, \eta) \in L$.

$$
\int_{L \backslash V}\|F(\dot{v})\|^{2} d \dot{v}=\int_{I_{\lambda} \times I_{\mu}}\|F(\lambda, \mu)\|^{2} d \lambda d \mu<\infty .
$$

Given $\phi \in \mathcal{H}_{\mathcal{M}, q_{\mathcal{M}}}$ and a fixed element $\Omega \in H_{g}$, we put 


$$
\begin{aligned}
& E_{\phi}(\lambda, \mu):=\phi((\lambda, \mu, 0)), \quad \lambda, \mu \in \mathbb{R}^{(h, g)}, \\
& F_{\phi}(\lambda, \mu):=\phi([\lambda, \mu, 0]), \quad \lambda, \mu \in \mathbb{R}^{(h, g)}, \\
& F_{\Omega, \phi}(\lambda, \mu):=e^{-2 \pi i \sigma\left(\mathcal{M} \lambda \Omega^{t} \lambda\right)} F_{\phi}(\lambda, \mu), \quad \lambda, \mu \in \mathbb{R}^{(h, g)} .
\end{aligned}
$$

In addition, we put for $W=\lambda \Omega+\mu \in \mathbb{C}^{(h, g)}$,

$$
\vartheta_{\Omega, \phi}(W):=\vartheta_{\Omega, \phi}(\lambda \Omega+\mu):=F_{\Omega, \phi}(\lambda, \mu) .
$$

We observe that $E_{\phi}, F_{\phi}$ and $F_{\Omega, \phi}$ are functions defined on $V$ and $\vartheta_{\Omega, \phi}$ is a function defined on $\mathbb{C}^{(h, g)}$.

Proposition 4.1. If $\phi \in \mathcal{H}_{\mathcal{M}, q_{\mathcal{M}}},(\xi, \eta) \in L$ and $(\lambda, \mu) \in V$, then we have the formulas

$$
\begin{gathered}
E_{\phi}(\lambda+\xi, \mu+\eta)=e^{2 \pi i \sigma\left\{\mathcal{M}\left(\xi^{t} \eta+\lambda^{t} \eta-\mu^{t} \xi\right)\right\}} E_{\phi}(\lambda, \mu) . \\
F_{\phi}(\lambda+\xi, \mu+\eta)=e^{-4 \pi i \sigma\left(\mathcal{M} \xi^{t} \mu\right)} F_{\phi}(\lambda, \mu) . \\
F_{\Omega, \phi}(\lambda+\xi, \mu+\eta)=e^{-2 \pi i \sigma\left\{\mathcal{M}\left(\xi \Omega^{t} \xi+2 \lambda \Omega^{t} \xi+2 \mu^{t} \xi\right)\right\}} F_{\Omega, \phi}(\lambda, \mu) .
\end{gathered}
$$

If $W=\lambda \Omega+\eta \in \mathbb{C}^{(h, g)}$, then we have

$$
\vartheta_{\Omega, \phi}(W+\xi \Omega+\eta)=e^{-2 \pi i \sigma\left\{\mathcal{M}\left(\xi \Omega^{t} \xi+2 W^{t} \xi\right)\right\}} \vartheta_{\Omega, \phi}(W) .
$$

Moreover, $F_{\phi}$ is an element of $\mathbf{H}_{\mathcal{M}, q_{\mathcal{M}}}$.

Proof. We note that

$$
(\lambda+\xi, \mu+\eta, 0)=\left(\xi, \eta,-\xi^{t} \mu+\eta^{t} \lambda\right) \circ(\lambda, \mu, 0) .
$$

Thus we have

$$
\begin{aligned}
E_{\phi}(\lambda+\xi, \mu+\eta) & =\phi((\lambda+\xi, \mu+\eta, 0)) \\
& =\phi\left(\left(\xi, \eta,-\xi^{t} \mu+\eta^{t} \lambda\right) \circ(\lambda, \mu, 0)\right) \\
& =e^{2 \pi i \sigma\left\{\mathcal{M}\left(\xi^{t} \eta+\lambda^{t} \eta-\mu^{t} \xi\right)\right\}} \phi((\lambda, \mu, 0)) \\
& =e^{2 \pi i \sigma\left\{\mathcal{M}\left(\xi^{t} \eta+\lambda^{t} \eta-\mu^{t} \xi\right)\right\}} E_{\phi}(\lambda, \mu) .
\end{aligned}
$$

This proves Formula (4.10). We observe that

$$
[\lambda+\xi, \mu+\eta, 0]=\left(\xi, \eta,-\xi^{t} \mu-\mu^{t} \xi-\eta^{t} \xi\right) \circ[\lambda, \mu, 0] .
$$


Thus we have

$$
\begin{aligned}
F_{\phi}(\lambda+\xi, \mu+\eta)= & ([\lambda+\xi, \mu+\eta, 0]) \\
= & e^{-2 \pi i \sigma\left\{\mathcal{M}\left(\xi^{t} \mu+\mu^{t} \xi+\eta^{t} \xi\right)\right\}} \\
& \times e^{2 \pi i \sigma\left(\mathcal{M} \xi^{t} \eta\right)} \phi([\lambda, \mu, 0]) \\
= & e^{-4 \pi i \sigma\left(\mathcal{M} \xi^{t} \mu\right)} \phi([\lambda, \mu, 0]) \\
= & e^{-4 \pi i \sigma\left(\mathcal{M} \xi^{t} \mu\right)} F_{\phi}(\lambda, \mu) .
\end{aligned}
$$

This proves Formula (4.11). According to (4.11), we have

$$
\begin{aligned}
F_{\Omega, \phi}(\lambda+\xi, \mu+\eta)= & e^{-2 \pi i \sigma\left\{\mathcal{M}(\lambda+\xi) \Omega^{t}(\lambda+\xi)\right\}} F_{\phi}(\lambda+\xi, \mu+\eta) \\
= & e^{-2 \pi i \sigma\left\{\mathcal{M}(\lambda+\xi) \Omega^{t}(\lambda+\xi)\right\}} \\
& \times e^{-4 \pi i \sigma\left(\mathcal{M} \xi^{t} \mu\right)} F_{\phi}(\lambda, \mu) \\
= & e^{-2 \pi i \sigma\left\{\mathcal{M}\left(\xi \Omega^{t} \xi+2 \lambda \Omega^{t} \xi+2 \mu^{t} \xi\right)\right\}} \\
& \times e^{-2 \pi i \sigma\left(\mathcal{M} \lambda \Omega^{t} \lambda\right)} F_{\phi}(\lambda, \mu) \\
= & e^{-2 \pi i \sigma\left\{\mathcal{M}\left(\xi \Omega^{t} \xi+2 \lambda \Omega^{t} \xi+2 \mu^{t} \xi\right)\right\}} F_{\Omega, \phi}(\lambda, \mu) .
\end{aligned}
$$

This proves Formula (4.12). Formula (4.13) follows immediately from Formula (4.12). Indeed, if $W=\lambda \Omega+\mu$ with $\lambda, \mu \in \mathbb{R}^{(h, g)}$, we have

$$
\begin{aligned}
\vartheta_{\Omega, \phi}(W+\xi \Omega+\eta) & =F_{\Omega, \phi}(\lambda+\xi, \mu+\eta) \\
& =e^{-2 \pi i \sigma\left\{\mathcal{M}\left(\xi \Omega^{t} \xi+2(\lambda \Omega+\mu)^{t} \xi\right)\right\}} F_{\Omega, \phi}(\lambda, \mu) \\
& =e^{-2 \pi i \sigma\left\{\mathcal{M}\left(\xi \Omega^{t} \xi+2 W^{t} \xi\right)\right\}} \vartheta_{\Omega, \phi}(W) .
\end{aligned}
$$

REMARK 4.2. The function $\vartheta_{\Omega, \phi}(W)$ is a theta function of level $2 \mathcal{M}$ with respect to $\Omega$ if $\vartheta_{\Omega, \phi}$ is holomorphic. For any $\phi \in \mathcal{H}_{\mathcal{M}, q_{\mathcal{M}}}$, the function $\vartheta_{\Omega, \phi}$ satisfies the well known transformation law of a theta function. In this sense, the lattice representation $\left(\pi_{\mathcal{M}, q_{\mathcal{M}}}, \mathcal{H}_{\mathcal{M}, q_{\mathcal{M}}}\right)$ is closely related to theta functions.

\section{REFERENCES}

[B] R. Berndt, Darstellungen der Heisenberggruppe und Thetafunktionen. Vorlesungsausarbeitung, Hamburg, 1988.

[C] P. Cartier, Quantum Mechanical Commutation Relations and Theta Functions, Proc. of Symp. Pure Mathematics, 9, Amer. Math. Soc., 1966, pp. 361-383. 
[F-C] G. Faltings and C.-L. Chai, Degeneration of Abelian Varieties, EMG, Band 22, SpringerVerlag, New York/Berlin, 1990.

[I] J. Igusa, Theta functions, Springer-Verlag, New York/Berlin, 1972.

[M] G. W. Mackey, Induced Representations of Locally Compact Groups I, Ann. of Math. 55 (1952), 101-139.

[N] Y. Namikawa, Toroidal Compactification of Siegel Spaces, Lect. Notes in Math. 812, Springer-Verlag, New York /Berlin, 1980.

[Y1] J.-H. Yang, Harmonic Analysis on the Quotient Spaces of Heisenberg Groups, Nagoya Math. J. 123 (1991), 103-117.

[Y2] - Harmonic Analysis on the Quotient Spaces of Heisenberg Groups, II, J. Number Theory 49 (1994), 63-72.

[Y3] - A decomposition theorem on differential polynomials of theta functions of high level, Japanese J. Math., Math. Soc. Japan, New Series 22 (1996), 37-49.

[Y4] , The Siegel-Jacobi Operator, Abh. Math. Sem. Univ. Hamburg 63 (1993), 135146.

[Y5] Remarks on Jacobi forms of higher degree, Proc. of the 1993 Workshop on Automorphic Forms and Related Topics, PIMS (1993), 33-58.

[Y6] - Singular Jacobi Forms, Trans. Amer. Math. Soc. 347 (1995), 2041-2049.

[Y7] Construction of Vector-Valued Modular Forms from Jacobi Forms, Canadian J. Math. 47 (1995), 1329-1339.

[Y8] - A geometrical theory of Jacobi forms of higher degree, Proc. of Symposium on Hodge Theory and Algebraic Geometry (edited by Tadao Oda), Sendai, Japan (1996), 125-147.

[Z] C. Ziegler, Jacobi Forms of Higher Degree, Abh. Math. Sem. Univ. Hamburg 59 (1989), 191-224.

Max-Planck Institut für Mathematik

Gottfried-Claren-Strasse 26

D-53225 Bonn

Germany

The present address is

Department of Mathematics

Inha University

Inchon 402-751

Republic of Korea

E-MAIL : JHYANG@INHA.AC.KR 